\title{
Lung Ultrasound for Early Diagnosis of Ventilator-Associated Pneumonia
}

\author{
AHMED A. ABD EL-WAHED, M.Sc.; THANAA M. AL-NOMANY, M.D.; \\ AHMED A. ABD EL-HAFEZ, M.D. and AHMED D. ABO HAMAR, M.D. \\ The Department ofAnesthesiology, Surgical Intensive Care and Pain Medicine, Faculty of Medicine, Tanta University
}

\begin{abstract}
Background: Lung Ultrasound (LUS) has important role in diagnosis of different lung diseases so it can be used in diagnosis and early detection of Ventilator-Associated Pneumonia (VAP).
\end{abstract}

Aim of Study: Our aim is to evaluate the sensitivity and the specificity of lung ultrasound for early diagnosis of ventilator-associated pneumonia compared to chest X-ray.

Patients and Methods: This study was carried out on 100 patients divided into two Groups (A \& B), each one included 50 adult male and female patients with suspected VAP. In Group A (LUS), we searched for lung ultrasound findings as subpleural consolidation, lobar consolidation, and dynamic arborescent/linear air bronchogram while in Group B (CXR), we searched for chest X-ray findings as lung infiltrates and air bronchogram. In both groups, Endotracheal Aspirates (EA) was collected for direct gram stain examination (EAgram) and culture (EAquant). LUS findings were analyzed in scores as the clinical-LUS score (Ventilator-associated Pneumonia Lung Ultrasound Score [VPLUS]) which was calculated as follows: $\geq 2$ areas with subpleural consolidations, 1 point; $\geq 1$ area with dynamic arborescent/linear air bronchogram, 2 points; and purulent EA, 1 point. Positive direct gram stain examination (EAgram) or positive culture (EAquant) which had 2 points were added to VPLUS to be VPLUS EAgram and VPLUS EAquant.

Results: The sensitivity and the specificity of lung ultrasound findings in Group A (LUS) were higher than chest Xray findings Group $B(C X R)$ as presence of ultrasound signs in Group A (LUS) (lobar/hemilobar consolidations, dynamic air bronchogram, subpleural consolidations) separate or combined gave us sensitivity $97 \%$, lobar or hemilobar consolidations had sensitivity $94 \%$, presence of dynamic air bronchogram or subpleural consolidations gave us sensitivity $94 \%$, VPLUS-EAquant $\geq 3$ gave us sensitivity $94 \%$. The best specificity was found also in Group A as (air bronchogram + subpleural consolidations + positive culture or positive gram stain examination) gave us the highest specificity $100 \%$, combination of (dynamic air bronchogram and subpleural consolidations) gave us high specificity $94 \%$, combination of (lobar/hemilobar consolidations, dynamic air bronchogram

Correspondence to: Dr. Ahmed A. Abd El-Wahed, E-Mail: ahmed127969@med.tanta.edu.eg. and subpleural consolidations) gave us also high specificity $94 \%$, (VPLUS-EAquant $\geq 4$, VPLUS-EAgram $\geq 4$ and VPLUS $\geq 3$ ) had specificity $94 \%$. On the other hand, signs of chest $\mathrm{X}$-ray in Group B had lower sensitivity and specificity compared to lung ultrasound in Group A as chest X-ray infiltrates gave us sensitivity $53 \%$, specificity $25 \%$, air bronchogram had sensitivity $33 \%$, specificity $40 \%$, presence of (chest Xray infiltraes, air bronchogram) separate or combined gave us sensitivity $57 \%$, specificity $25 \%$.

Conclusion: The sensitivity and specificity of lung ultrasound were higher than chest xray, so lung ultrasound is better than chest X-ray for early diagnosis of VAP.

Key Words: Lung ultrasound - Chest X-ray - VentilatorAssociated Pneumonia (VAP).

\section{Introduction}

VENTILATOR-Associated Pneumonia (VAP) is a common respiratory disease which occurs 48 hours or more on Mechanical Ventilation (MV), associated with increased mortality and morbidity. There are two types of VAP: (A) Early onset VAP which occurs within the first 4 days of mechanical ventilation [1]. (B) Late onset VAP which happens after day 4 and is more frequently due to MultidrugResistant pathogens (MDR) [2] .

VAP suspected by presence of a new lung infiltrate in chest radiographs after admission, with at least two of the following clinical signs and symptoms: Purulent tracheal secretions, body temperature $\left(\geq 38.5^{\circ} \mathrm{C}\right.$ or $\left.\leq 36.5^{\circ} \mathrm{C}\right)$, leucocytosis $(>11,000$ cells $\left./ \mathrm{mm}^{3}\right)$ or leucopenia $\left(<4,000\right.$ cells $\left./ \mathrm{mm}^{3}\right), \mathrm{PaO}_{2}$ to $\mathrm{FiO}_{2}$ ratio $<300 \mathrm{mmHg}$ with no evidence of acute respiratory distress syndrome $[3,4]$.

Diagnosis of VAP includes clinical data, chest xray, culture, gram stain examination and lung ultrasound. Culture from endotracheal tube is the gold standard for diagnosis of VAP. Chest X-ray used for diagnosis of VAP by finding new lung 
infiltrates which may bilateral scattered, lobar or hemilobar [5].

Also lung ultrasound used in diagnosis of VAP. Lobar/hemilobar consolidations, sub-pleural consolidations, dynamic air bronchogram are the sonographic signs of VAP [6] . Prevention of VAP can be done by some ways as limiting exposure to mechanical ventilation, preferring non-mechanical ventilation when possible, reducing airways colonization by oral care decontamination using chlorhexidine, or preventing aspiration (e.g. by nursing in the semi-recumbent position, or maintaining a sufficient cuff pressure), daily sedation hold, strict hand hygiene with alcohol especially before managing the airways, prevention of biofilm formation in the lumen of endotracheal tube and around the cuff $[\mathbf{7 , 8}]$.

Treatment involves identifying the causal germs and active antibiotic therapy. Any delay in starting antibiotics in severe sepsis increases mortality, therefore the need for early detection of VAP $[9,10]$ In this study, we evaluated the role of lung ultrasound for early detection of ventilator-associated pneumonia in comparison to chest X-ray.

\section{Material and Methods}

This study was carried out in Tanta University Hospitals at Surgical Intensive Care Unit (SICU) from June 2017 to May 2018 after approval from Institutional Ethical Committee, all data of patients were confidential with secret codes and private file for each patient, also an informed consent was obtained from every patient participating in this study that included 100 patients divided into two Groups (A \& B), each one included 50 adult male and female patients with suspected VAP ranged from 18 to 70 years old.

\section{Inclusion criteria:}

The study included the patients with suspected VAP. The duration of MV differed from one patient to another and so time of suspicion of VAP. Patients put on MV due to different causes as intra cranial haemorrhage, brain tumor, polytrauma, intestinal obstruction, abdominal exploration. Vital data as temperature, blood pressure, heart rate, oxygen saturation were measured regulary as routine assessment in ICU.

Routine investigations as $\mathrm{CBC}$, eletrolyte, $\mathrm{ABG}$ and others were done, assessed in regular way. Also chest X-ray and culture from endotracheal tube were routine investigations in ICU. Clinical suspicion of VAP was based on the classical criteria as: Patient on MV > $48 \mathrm{~h}$, two or more of the fol- lowing criteria: Fever $\left(>38.5^{\circ} \mathrm{C}\right)$ or hypothermia $\left(<36.5^{\circ} \mathrm{C}\right)$, leukocytosis $(>11,000 / \mathrm{ml})$ or leukopenia $(<4000 / \mathrm{ml})$, purulent tracheal secretions, Pao 2/Fio2 $(<300 \mathrm{mmHg})[4]$.

\section{Exclusion criteria:}

- Patients $(<18->70)$ years old.

- Patients who already diagnosed VAP or had any clinical suspicion in $<48 \mathrm{~h}$ of start of MV.

\section{Group classification:}

Group A: This group included 50 adult male and female patients at time VAP was suspected, lung ultrasound with direct gram stain examination and culture from endotracheal aspirate were done for each patient when VAP was suspected. Endotracheal aspirate was collected through sterile catheter from endotracheal tube then submitted to direct gram stain examination (EAgram) and culture (EAquant), EAgram was considered positive if any bacteria was visualized after gram stain testing on tracheal secretions, EAquant was positive and confirmed diagnosis of VAP when ( $>1$ microorganism with a concentration $>10^{4} \mathrm{CFU} / \mathrm{ml}$ ) [11]. The ultrasound probe that was used in Lung Ultrasound (LUS) is $(3-5 \mathrm{MHz})$ deep convex probe that allowed good visualization of the lung.

Examination was done in supine position in six areas (superior and inferior areas in the anterior, lateral, posterior fields using parasternal, paravertebral, anterior and posterior axillary lines as landmarks, with transverse line between parasternal and paravertebral line through the nipple) Fig. (1). The lateral position was used for posterior lung surface examination and the probe put vertically on the chest tilting it to get good image Fig. (2).

The following ultrasound findings were collected:

1- Small subpleural consolidations (echo-poor regions $>0.5 \mathrm{~cm}$ in diameter).

2- Lobar/hemilobar consolidations defined by a tissue-like pattern.

3- Dynamic linear or arborescent air bronchogram within lobar/hemilobar consolidations (air entrapped within bronchi with simultaneous movement with inspiration) Fig. (4).

Ultrasound findings were collected together in a score called (Ventilator-associated Pneumonia Lung Ultrasound Score) (VPLUS) [12] that was as followed:

$->2$ areas with subpleural consolidations, 1 point.

$->1$ area with dynamic linear or arborescent air bronchogram, 2 points. 
- Purulent Endotracheal Aspirate (EA), 1 point.

Also ultrasound findings and microbiological findings were collected together in scores called (ventilator-associated pneumonia lung ultrasound direct gram stain examination \& culture score) (VPLUS EAgram \& VPLUS EAquant) [12] that were as followed:

$-\geq 2$ areas with subpleural consolidations, 1 point.

$-\geq 1$ area with dynamic linear or arborescent air bronchogram, 2 points.

- Purulent Endotracheal Aspirate (EA), 1 point.

Positive direct gram stain examination or culture (EAgram/EAquant), 2 points.

Sensitivity and specificity of VPLUS, VPLUS EAgram, VPLUS EAquant scores were measured to help in early diagnosis of VAP.

Group B: This group also included 50 adult male and female patients with suspected VAP, chest $\mathrm{X}$-ray was done for every patient in this group with also direct gram stain examination (EAgram) \& culture (EAquant) from Endotracheal Aspirate (EA) at time VAP was suspected. New chest Xray was done to the patients who had chest xray done before clinical suspicion. EA was collected through sterile catheter passed through endotracheal tube, EAgram was considered positive if any bacteria was visualized after gram stain testing on tracheal secretions, EAquant was positive and confirmed diagnosis of VAP when ( $\geq 1$ microorganism with a concentration $\geq 104 \mathrm{CFU} / \mathrm{ml}$ ).

The most common findings of chest X-ray in VAP were lung infiltrates or patches that involve one lobe or more or may be scattered all over the lung Fig. (5). Also there were other findings as air bronchograms, para pneumonic effusion, silhouette sign (loss of normal borders between thoracic structures). Culture and gram stain examination results were appeared within 2-4 days, their results were correlated with the findings of lung ultrasound and chest X-ray which were done at time of sucpicion.

Statistical presentation and analysis was conducted by SPSS V.24. Results were expressed as means \pm Standard Deviation (SD). Sensitivity and Specificity were calculated for LUS signs (lobar/ hemilobar consolidation,dynamic linear/arborescent air bronchograms, and subpleural consolidation), chest X-ray signs, clinical (purulent secretions) and for microbiologic (EA).

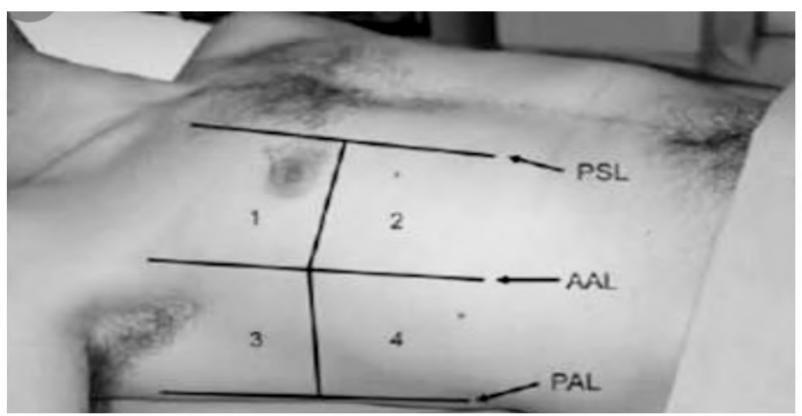

Fig. (1): Anterior zone examination.

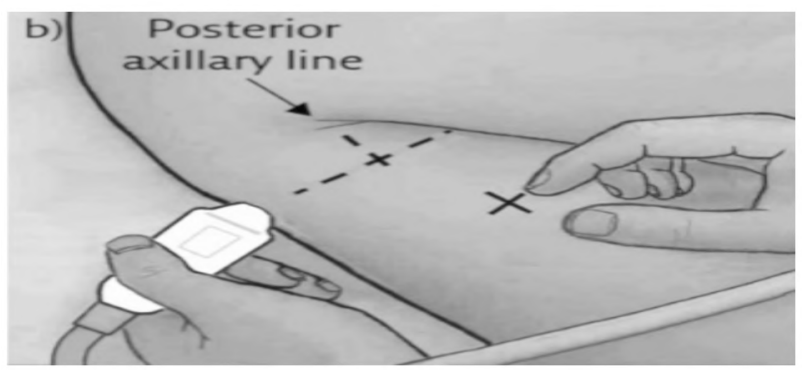

Fig. (2): Posterior zone examination.

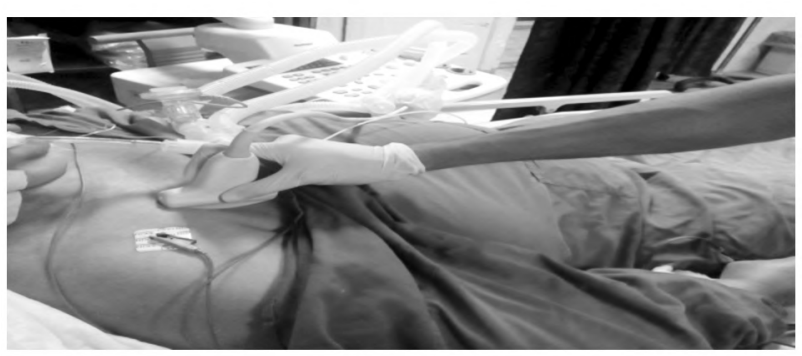

Fig. (3): Lung ultrasound examination.

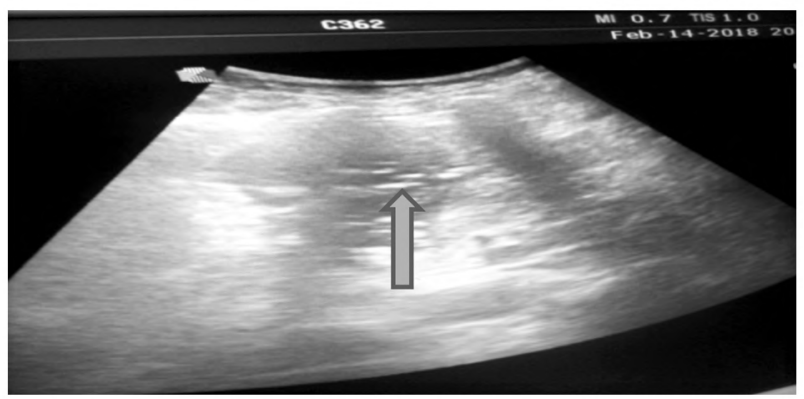

Fig. (4): Air bronchogram inside lobar consolidation.

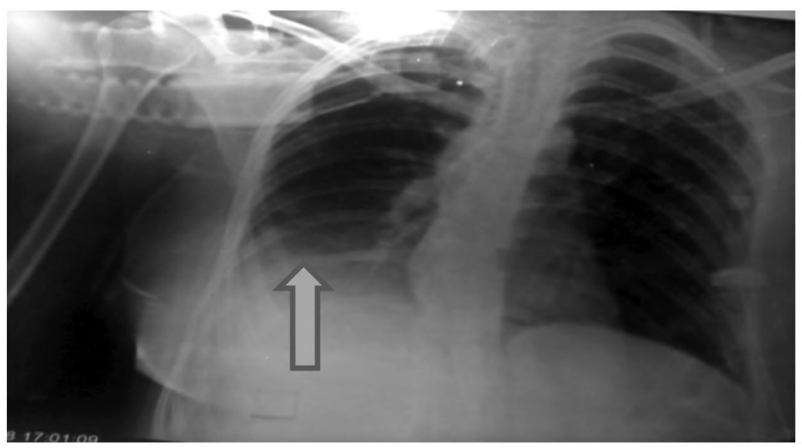

Fig. (5): Chest X-ray infiltrate. 


\section{Results}

Our results showed that there was no significant difference between both group regarding demographic data (age, BMI \& sex), duration of MV at time of suspicion of VAP. Pseudomonas aeruginosa was the most common organism in Group A while Escherichia coli was the most common one in Group B. The first 33 patients were VAP patients in Group A (LUS) while first 30 patients were VAP patients in Group B (CXR).

If we search for the best sensitivity we found it in Group A, presence of ultrasound signs in Group A (LUS) (lobar/hemilobar consolidations, dynamic air bronchogram, subpleural consolidations) separate or combined gave us sensitivity $97 \%$, lobar or hemilobar consolidations had sensitivity $94 \%$, presence of dynamic air bronchogram or subpleural consolidations gave us sensitivity $94 \%$, VPLUS-EAquant $\geq 3$ gave us sensitivity $94 \%$, VPLUS-EAgram $\geq 3$ gave us sensitivity $85 \%$, subpleural consolidation $\geq 1$ had sensitivity $82 \%$.

The best specificity found also in Group A as (air bronchogram + subpleural consolidations + positive culture or positive gram stain examination) gave us the highest specificity $100 \%$, also combination between dynamic air bronchogram $\geq 1$ or 2 and positive culture or gram stain examination had high specificity $94 \%$, combination of (dynamic air bronchogram and subpleural consolidations) gave us high specificity $94 \%$, combination of (lobar/ hemilobar consolidations, dynamic air bronchogram and subpleural consolidations) gave us also high specificity $94 \%$, subpleural consolidation $\geq 2$ and positive gram stain examination (EAgram) gave us specificity $94 \%$, (VPLUS-EAquant $\geq 4$, VPLUSEAgram $\geq 4$ and VPLUS $\geq 3$ ) had specificity $94 \%$, (VPLUS-EAgram $\geq 3$ and VPLUS $\geq 2$ ) had specificity $82 \%$, subpleural consolidations $\geq 1$ and positive gram stain examination or culture had specificity $88 \%$, dynamic air bronchogram $\geq 2$ alone had high specificity $88 \%$, dynamic air bronchogram $\geq 1$ also had high specificity $82 \%$.

On the other hand, signs of chest X-ray in Group B had lower sensitivity and specificity compared to lung ultrasound in Group A as chest xray infiltrates gave us sensitivity $53 \%$, specificity $25 \%$, air bronchogram had sensitivity $33 \%$, specificity $40 \%$, presence of (chest xray infiltraes, air bronchogram) separate or combined gave us sensitivity $57 \%$, specificity $25 \%$, also if we compared each sign in both groups with another as lobar consolidations to chest X-ray infiltrates and air bronchogram in both groups we found that lung ultrasound had better results.

According to the above mentioned results, Group A, sensitivity of lung ultrasound reachs $97 \%$ and specificity reachs $94 \%$, Group B, sensitivity of chest X-ray reachs $57 \%$ while specificity reachs $40 \%$, so lung ultrasound is better than chest X-ray for early diagnosis of VAP.

Area Under the Curve (AUC) was (0.932) for VPLUS, (0.878) for VPLUS-EAquant and (0.948) for VPLUS-EAgram.

Table (1): Demographic data in both groups.

\begin{tabular}{llll}
\hline & $\begin{array}{c}\text { Group A } \\
\text { (LUS) }\end{array}$ & $\begin{array}{c}\text { Group B } \\
(\mathrm{CXR})\end{array}$ & $\begin{array}{c}p \text { - } \\
\text { value }\end{array}$ \\
\hline - Age $(\mathrm{year}){ }^{2}$ ). & $37.9 \pm 13.7$ & $38.6 \pm 10.8$ & 0.801 \\
- BMI $\left(\mathrm{kg} / \mathrm{m}^{2}\right)$ & $25.1 \pm 5.7$ & $26.6 \pm 6.1$ & 0.224 \\
- Sex $(\mathrm{M} / \mathrm{F})$. & $30 / 20$ & $28 / 22$ & 0.839 \\
- Duration of MV at time of & $4.7 \pm 1.6$ & $5 \pm 2.1$ & 0.521 \\
$\quad$ suspicion of VAP (days). & & & \\
\hline$p$-value significant if $<0.05$. & & &
\end{tabular}

Table (2): Diagnostic value of VPLUS, VPLUS-EAquant, VPLUS-EAgram.

\begin{tabular}{lccccc}
\hline & Sensitivity & Specificity & PPV & NPV & N \\
\hline VPLUS $\geq 2$ & $79 \%$ & $82 \%$ & $90 \%$ & $67 \%$ & 29 \\
VPLUS $\geq 3$ & $64 \%$ & $94 \%$ & $95 \%$ & $57 \%$ & 22 \\
VPLUS EAquant $\geq 3$ & $94 \%$ & $76 \%$ & $89 \%$ & $87 \%$ & 35 \\
VPLUS EAquant $\geq 4$ & $73 \%$ & $94 \%$ & $96 \%$ & $64 \%$ & 25 \\
VPLUS EAgram $\geq 3$ & $85 \%$ & $82 \%$ & $90 \%$ & $74 \%$ & 31 \\
VPLUS EAgram $\geq 4$ & $67 \%$ & $94 \%$ & $96 \%$ & $59 \%$ & 23 \\
\hline
\end{tabular}

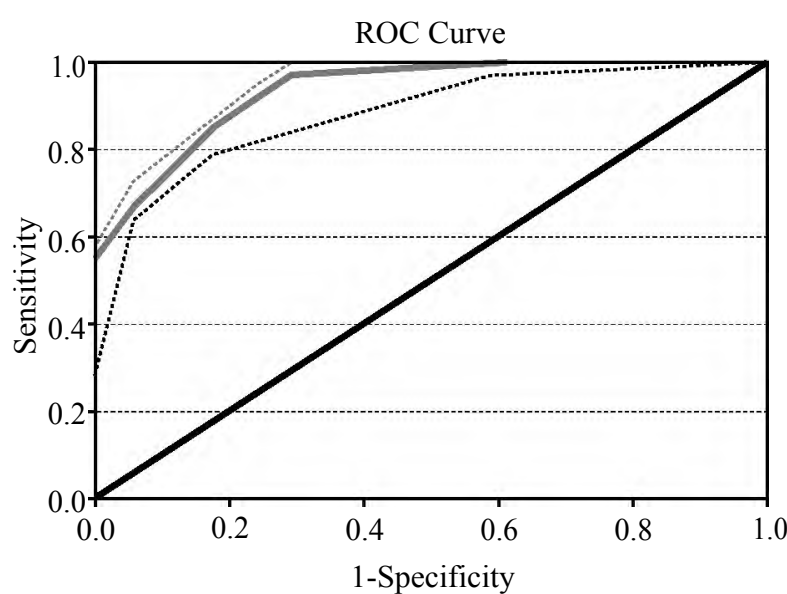

Diagonal segments are produced by ties

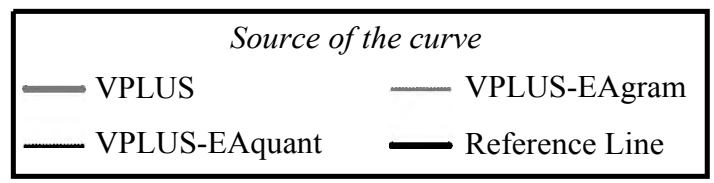

Fig. (6): Receiver Operating Characteristic (ROC) curves for VPLUS, VPLUS-EAquant, VPLUS-EAgram in Group A. 
Table (3): Findings of lung ultrasound in Group A.

\begin{tabular}{|c|c|c|c|c|c|c|c|c|}
\hline & $\begin{array}{c}\text { Lobar/hemi } \\
\text { lobar } \\
\text { consolidation }\end{array}$ & $\begin{array}{c}\text { Dynamic air } \\
\text { bronchogram } \\
\geq 1\end{array}$ & $\begin{array}{c}\text { Dynamic air } \\
\text { bronchogram } \\
>2\end{array}$ & $\begin{array}{c}\text { Subpleural } \\
\text { consolidation } \\
\geq 1\end{array}$ & $\begin{array}{c}\text { Subpleural } \\
\text { consolidation } \\
>2\end{array}$ & $\begin{array}{l}\text { Culture } \\
\text { (EAquant) }\end{array}$ & $\begin{array}{l}\text { Gram stain } \\
\text { (EAgram) }\end{array}$ & $\begin{array}{l}\text { Purulent } \\
\text { secretions }\end{array}$ \\
\hline 1 & + & + & + & + & + & + & + & \\
\hline 2 & + & + & + & + & + & + & + & + \\
\hline 3 & + & + & + & + & - & + & + & + \\
\hline 4 & + & & - & + & + & - & + & + \\
\hline 5 & + & + & + & + & + & + & + & \\
\hline 6 & + & - & - & - & - & + & - & + \\
\hline 7 & + & + & + & + & - & + & + & - \\
\hline 8 & + & + & + & + & + & + & + & + \\
\hline 9 & + & + & + & + & + & + & + & + \\
\hline 10 & + & + & + & + & + & + & + & - \\
\hline 11 & + & - & - & + & + & + & - & + \\
\hline 12 & + & + & - & + & - & + & + & + \\
\hline 13 & + & + & - & + & + & + & + & + \\
\hline 14 & + & - & & + & + & + & - & + \\
\hline 15 & + & + & & + & + & + & + & - \\
\hline 16 & + & + & + & + & + & + & + & + \\
\hline 17 & + & + & + & - & - & - & + & + \\
\hline 18 & + & + & - & + & + & + & - & + \\
\hline 19 & + & - & - & + & + & + & + & - \\
\hline 20 & + & + & - & + & + & - & + & + \\
\hline 21 & + & + & + & & - & + & + & + \\
\hline 22 & + & - & - & + & - & + & + & + \\
\hline 23 & & + & - & & - & + & + & + \\
\hline 24 & + & + & - & + & + & + & - & + \\
\hline 25 & - & - & - & - & & + & + & + \\
\hline 26 & + & + & + & + & + & + & + & + \\
\hline 27 & + & - & - & + & - & + & + & + \\
\hline 28 & + & + & - & + & + & + & & - \\
\hline 29 & + & - & - & + & + & + & & + \\
\hline 30 & + & + & + & - & - & + & + & + \\
\hline 31 & + & - & - & + & + & + & + & - \\
\hline 32 & + & + & + & + & - & + & + & + \\
\hline 33 & + & - & - & + & - & + & + & - \\
\hline 34 & + & - & - & - & - & - & - & - \\
\hline 35 & - & - & - & - & - & - & - & - \\
\hline 36 & + & - & - & - & - & - & - & - \\
\hline 37 & - & - & - & - & - & - & - & - \\
\hline 38 & + & & & + & + & + & - & - \\
\hline 39 & - & & & - & - & & - & + \\
\hline 40 & + & + & + & - & - & - & + & \\
\hline 41 & + & + & + & - & - & + & - & - \\
\hline 42 & + & - & - & & & - & - & + \\
\hline 43 & - & - & - & & & - & - & - \\
\hline 44 & + & - & - & + & + & - & + & - \\
\hline 45 & + & + & - & + & + & - & - & - \\
\hline 46 & - & - & & & & - & - & + \\
\hline 47 & + & & & + & & - & + & - \\
\hline 48 & + & & & + & + & + & & \\
\hline 49 & - & & & & & & & + \\
\hline 50 & + & & & & & & & \\
\hline Sensitivity & $94 \%$ & $67 \%$ & $42 \%$ & $82 \%$ & $61 \%$ & $91 \%$ & $79 \%$ & $73 \%$ \\
\hline Specificity & $35 \%$ & $82 \%$ & $88 \%$ & $71 \%$ & $76 \%$ & $82 \%$ & $82 \%$ & $76 \%$ \\
\hline PPV & $74 \%$ & $88 \%$ & $88 \%$ & $84 \%$ & $83 \%$ & $91 \%$ & $90 \%$ & $86 \%$ \\
\hline NPV & $75 \%$ & $56 \%$ & $44 \%$ & $67 \%$ & $50 \%$ & $82 \%$ & $67 \%$ & $59 \%$ \\
\hline $\mathrm{N}$ & 42 & 25 & 16 & 32 & 24 & 33 & 29 & 28 \\
\hline
\end{tabular}


Table (4): Combination between findings of lung ultrasound in Group A.

\begin{tabular}{|c|c|c|c|c|c|}
\hline & Sensitivity & Specificity & PPV & NPV & $\mathrm{N}$ \\
\hline Dynamic air bronchogram and subpleural consolidations. & $55 \%$ & $94 \%$ & $95 \%$ & $52 \%$ & 19 \\
\hline Dynamic air bronchogram or subpleural consolidations. & $94 \%$ & $59 \%$ & $82 \%$ & $83 \%$ & 38 \\
\hline Dynamic air bronchogram + subpleural consolidations and lobar/hemilobar consolidations. & $55 \%$ & $94 \%$ & $95 \%$ & $52 \%$ & 19 \\
\hline Dynamic air bronchogram or subpleural consolidations or lobar/hemilobar consolidations. & $97 \%$ & $35 \%$ & $74 \%$ & $86 \%$ & 43 \\
\hline Dynamic air bronchogram $>1$ and positive culture. & $61 \%$ & $94 \%$ & $95 \%$ & $55 \%$ & 21 \\
\hline Dynamic air bronchogram $>1$ and positive EAgram. & $58 \%$ & $94 \%$ & $95 \%$ & $53 \%$ & 20 \\
\hline Dynamic air bronchogram $>2$ and positive culture. & $39 \%$ & $94 \%$ & $93 \%$ & $44 \%$ & 14 \\
\hline Dynamic air bronchogram $>2$ and positive EAgram. & $42 \%$ & $94 \%$ & $93 \%$ & $46 \%$ & 15 \\
\hline Subpleural consolidations $>1$ and positive culture. & $76 \%$ & $88 \%$ & $93 \%$ & $65 \%$ & 27 \\
\hline Subpleural consolidations $>1$ and positive EAgram. & $64 \%$ & $88 \%$ & $91 \%$ & $56 \%$ & 23 \\
\hline Subpleural consolidations $>2$ and positive culture. & $55 \%$ & $88 \%$ & $90 \%$ & $50 \%$ & 20 \\
\hline Subpleural consolidations $>2$ and positive EAgram. & $42 \%$ & $94 \%$ & $93 \%$ & $46 \%$ & 15 \\
\hline Dynamic air bronchogram + subpleural consolidations and positive culture. & $52 \%$ & $100 \%$ & $100 \%$ & $52 \%$ & 17 \\
\hline Dynamic air bronchogram + subpleural consolidations and positive EAgram. & $45 \%$ & $100 \%$ & $100 \%$ & $49 \%$ & 15 \\
\hline
\end{tabular}

Table (5): Findings of chest X-ray in Group B.

\begin{tabular}{|c|c|c|c|c|c|c|c|}
\hline & $\begin{array}{c}\text { Chest X-ray } \\
\text { infiltrates }\end{array}$ & $\begin{array}{c}\text { Air } \\
\text { bronchogram }\end{array}$ & $\begin{array}{c}\text { Culture } \\
\text { (EAquant) }\end{array}$ & $\begin{array}{c}\text { Gram stain } \\
\text { examination } \\
\text { (EAgram) }\end{array}$ & $\begin{array}{c}\text { Purulent } \\
\text { secretions }\end{array}$ & $\begin{array}{c}\text { Chest X-ray } \\
\text { infiltrates or } \\
\text { air bronchogram }\end{array}$ & $\begin{array}{c}\text { Chest X-ray } \\
\text { infiltrates and } \\
\text { air bronchogram }\end{array}$ \\
\hline 1 & + & + & + & + & + & + & + \\
\hline 2 & + & & + & + & - & + & \\
\hline 3 & - & - & + & + & + & - & - \\
\hline 4 & + & + & + & + & - & + & + \\
\hline 5 & + & - & + & - & + & + & - \\
\hline 6 & - & - & + & + & + & - & - \\
\hline 7 & + & + & - & + & + & + & + \\
\hline 8 & + & - & + & - & + & + & - \\
\hline 9 & - & - & + & + & + & - & - \\
\hline 10 & + & + & + & + & - & + & + \\
\hline 11 & - & - & + & + & + & - & - \\
\hline 12 & + & + & - & + & + & + & + \\
\hline 13 & + & - & + & - & + & + & - \\
\hline 14 & - & - & + & + & + & - & - \\
\hline 15 & + & - & - & + & + & + & - \\
\hline 16 & + & + & + & - & + & + & + \\
\hline 17 & - & - & + & + & + & - & - \\
\hline 18 & - & - & + & + & + & - & - \\
\hline 19 & + & + & + & + & - & + & + \\
\hline 20 & - & - & + & + & + & - & - \\
\hline 21 & + & - & + & + & + & + & - \\
\hline 22 & - & - & + & + & + & - & - \\
\hline 23 & + & + & + & - & - & + & + \\
\hline 24 & - & - & + & + & - & - & - \\
\hline 25 & + & - & + & - & + & + & \\
\hline 26 & - & - & + & + & - & - & \\
\hline 27 & - & + & + & - & + & + & \\
\hline 28 & - & - & + & + & + & - & - \\
\hline 29 & + & + & - & + & + & + & + \\
\hline 30 & - & - & + & + & + & - & - \\
\hline 31 & + & + & - & + & + & + & + \\
\hline 32 & + & + & - & - & - & + & + \\
\hline 33 & + & - & + & - & - & + & - \\
\hline 34 & + & + & - & & & + & + \\
\hline 35 & + & - & + & & & + & - \\
\hline 36 & + & + & - & - & - & + & + \\
\hline 37 & - & - & - & + & + & - & - \\
\hline 38 & + & + & - & - & - & + & + \\
\hline 39 & - & - & + & - & - & - & - \\
\hline 40 & + & + & - & - & - & + & + \\
\hline 41 & - & - & - & + & & - & - \\
\hline 42 & + & + & - & - & & + & + \\
\hline 43 & + & + & - & - & - & + & + \\
\hline 44 & - & - & - & + & + & - & - \\
\hline 45 & + & + & & - & - & + & + \\
\hline 46 & + & - & & + & + & + & - \\
\hline 47 & + & + & - & - & - & + & + \\
\hline 48 & - & - & + & - & - & - & - \\
\hline 49 & + & + & - & - & & + & + \\
\hline 50 & + & + & & & + & + & + \\
\hline Sensitivity & $53 \%$ & $33 \%$ & $87 \%$ & $77 \%$ & $77 \%$ & $57 \%$ & $30 \%$ \\
\hline Specificity & $25 \%$ & $40 \%$ & $80 \%$ & $80 \%$ & $75 \%$ & $25 \%$ & $40 \%$ \\
\hline PPV & $52 \%$ & $45 \%$ & $87 \%$ & $85 \%$ & $82 \%$ & $53 \%$ & $43 \%$ \\
\hline NPV & $26 \%$ & $29 \%$ & $80 \%$ & $70 \%$ & $68 \%$ & $28 \%$ & $28 \%$ \\
\hline $\mathrm{N}$ & 31 & 22 & 30 & 27 & 28 & 32 & 21 \\
\hline
\end{tabular}




\section{Discussion}

VAP is a serious respiratory disease that increases the rate of morbidity and mortality in ICU. VAP was suspected when a new radiographic infiltrate developed in a patient with fever/hypothermia, leukocytosis/leukopenia, purulent tracheal secretions, and impaired oxygenation. Many non infectious processes can cause fever and pulmonary infiltrates so these clinical signs are not specific only to VAP, therefore the need for early detection and early administration of antibiotics.

Lung ultrasound had advantages in diagnosis of VAP as it is a bedside noninvasive technique, easily available, no exposure to radiation, not cost much, safe in pregnant women, highly accurate, but it had some limitations as LUS is operator dependent and requires a trained physician, some patients may be difficult to examine by using LUS (eg, obese individuals, patients with subcutaneous emphysema or large thoracic dressings). Sonographic signs of VAP were lobar/hemilobar consolidations, subpleural consolidations, dynamic air bronchogram or fluid bronchogram.

Chest X-ray used as a routine tool for diagnosis of VAP by finding new lung infiltrates which may bilateral scattered, lobar or hemilobar but it had disadvantages as radiation, difficult in transporting device and critically ill patients, cost, not easily available, not highly accurate and not safe in pregnant women, sometimes bad quality of films. Culture from endotracheal tube is the gold standard for diagnosis of VAP which is positive when ( $>1$ microorganism with a concentration $>10^{4} \mathrm{CFU} / \mathrm{ml}$ ) but it needed 2 to 4 days to appear. Mechanism of VAP came from migration of microorganisms through endotracheal tube by positive-pressure MV.

The sensitivity and specificity of LUS signs were high especially when combined with microbiological findings as (air bronchogram + subpleural consolidations + positive culture or positive gram stain examination) gave us the highest specificity $100 \%$ and PPV $100 \%$, presence of ultrasound signs (lobar/hemilobar consolidations, dynamic air bronchogram, subpleural consolidations) separate or combined gave us sensitivity $97 \%$, lobar or hemilobar consolidations had sensitivity 94\% found in most patients, presence of dynamic air bronchogram or subpleural consolidations gave us sensitivity 94\%, combination of (dynamic air bronchogram and subpleural consolidations) gave us high specificity $94 \%$, combination between dynamic air bronchogram $>1$ or 2 and positive culture or gram stain examination had high specificity $94 \%$, subpleural consolidation $>2$ and positive gram stain examination (EAgram) gave us specificity $94 \%$, subpleural consolidations $>1$ and positive gram stain examination or culture had specificity $88 \%$, also VPLUS-EAquant $>3$ gave us sensitivity $94 \%$ and (VPLUS-EAquant $>4$, VPLUS-EAgram $>4$ and VPLUS $>3$ ) had high specificity $94 \%$ while chest xray in Group B (CXR) had lower sensitivity and specificity compared to lung ultrasound in Group A.

In agreement with our results, Mongodi et al., [12], assessed the accuracy of lung ultrasound in 99 patients with suspected VAP, lobar/hemi lobar consolidation occurred universally in patients without VAP, with sensitivity $93 \%$ and specificity was 0 . One or more areas with a small subpleuralconsolidation had a sensitivity of $81 \%$ and a specificity of $41 \%$, whereas one or more areas with a consolidation and dynamic air bronchograms had a sensitivity of $44 \%$ and a specificity of $81 \%$. The specificity of these signs increased when they were present in a greater number of areas, VPLUSEAgram $>4$ had a sensitivity of $48 \%$ and a specificity of $97 \%$, VPLUS-EAgram $>3$ had a sensitivity of 78 up to $88 \%$ and a specificity of 77 up to $90 \%$, VPLUS-EAquant $>4$ had a sensitivity of $57 \%$ and a specificity of $96 \%$, VPLUS-EAquant $>3$ had a sensitivity of 83 up to $92 \%$ and a specificity of 79 up to $92 \%$.

In agreement with our results, Cortellaro et al., [13],prospective study was done on 120 patients, pneumonia suspected by clinical criteria as leukocytosis, leucopenia, fever hypoxia, new infiltrate in chest xray, diagnosis by ultrasound by detection of consolidations and dynamic air bronchogram. This gave us sensitivity of $99 \%$ and specificity of 95\%, makes ultrasound better than X-ray for diagnosis of pneumonia.

Similarly, Lichtenstein et al., [14], prospective study that was done on 260 patients, suspicion based on clinical picture and new CXR infiltrates, using lung ultrasound for detection of dynamic air bronchogram in mechanically ventilated patients with pneumonia. The sensitivity reported in this study was $89 \%$ and the specificity was $94 \%$ in diagnosing VAP. Also in agreement with our results, Berlet et al., [15], performed daily LUS for at least 5 days in 57 patients and assessed consolidations, dynamic air bronchogram, fluid bronchogram. Lung ultrasound had a sensitivity of $92 \%$ and a specificity of $65 \%(40-80 \%)$.

In disagreement with our results, Corradi et al., [16], prospective study was done on 35 patients in 
ED, pneumonia suspected clinically and chest Xray infiltrates, ultrasound diagnosis of pneumonia done by finding consolidations and air bronchograms. The sensitivity of lung ultrasound was $57 \%$ that is much lower than ours, specificity up to $86 \%$. This disagreement may be due to number of patients in this study was lower than our study, may be also because inclusion involved community acquired not only ventilator associated pneumonia.

In disagreement with our results, Gatt et al., [17], this study was done on large number of patients 507 in ED, chest radiology was done for detection of any abnormalities such as consolidations, pleural effusion, congestion or any abnormalities, the sensitivity of consolidations by chest $\mathrm{X}$-ray was $65 \%$ and specificity was $95 \%$, this comes with disagreement with our results which chest X-ray has lower results in sensitivity and specificity, this may be due to large number of patients in this study, also not specific for studying consolidations only but any other abnormalities as well.

Also in disagreement with our results, Zagli et al., [18], retrospectively investigated the accuracy of alveolar consolidation in a comprehensive LUS examination. Sonographic consolidation had a sensitivity of $59 \%$ and a specificity of $84 \%$, our results consolidations have higher sensitivity reached to $94 \%$. It may be due to Zagli worked on more patients 221 . Also this study assessed the accuracy of LUS, when used in conjunction with Clinical Pulmonary Infection Score (CPIS) and Procalcitonin.

Xirouchaki et al., [19] prospective study was done on 42 mechanically ventilated patients in ICU. Chest X-ray, lung ultrasound, CT scan were done for diagnosis of consolidation, interstitial syndrome, pneumothorax and pleural effusion. According to consolidations by lung ultrasound, sensitivity was $100 \%$, specificity was $78 \%$ while by chest X-ray, sensitivity was $38 \%$, specificity was $89 \%$, this study comes with agreement with our study in high sensitivity of lung ultrasound, lower sensitivity of chest X-ray but it comes with disagreement with our study in high specificity of chest X-ray and became higher than lung ultrasound.

\section{Conclusion:}

Lung ultrasound has several advantages over chest X-ray. It has high diagnostic accuracy in diagnosis of VAP. Its sensitivity and specificity higher than chest $\mathrm{X}$-ray, so lung ultrasound is better than chest X-ray for early diagnosis of VAP.

\section{Conflicts of interest:}

No conflicts of interest declared.

Authors' contributions:

All authors had equal role in design, work, statistical analysis and manuscript writing.

\section{References}

1- JOSEPH N.M., SISTLA S., DUTTA T.K., BADHE A.S and PARIJA S.C.: Ventilator-associated pneumonia: A review. European Journal of Internal Medicine, 21 (5): 360-8, 2010.

2- ANAND N. and KOLLEF M.H.: Editors. The alphabet soup of pneumonia: CAP, HAP, HCAP, NHAP, and VAP. Seminars in respiratory and critical care medicine, (C) Thieme Medical Publishers, 2009.

3- SOCIETY A.T. and AMERICA I.D.S.O.: Guidelines for the management of adults with hospital-acquired, ventilator-associated, and healthcare-associated pneumonia. American Journal of Respiratory and Critical Care Medicine, 171 (4): 388, 2005.

4- CHASTRE J. and FAGON J.Y.: Ventilator-associated pneumonia. American Journal of Respiratory and Critical Care Medicine, 165 (7): 867-903, 2002.

5- LICHTENSTEIN D., GOLDSTEIN I., MOURGEON E., CLUZEL P., GRENIER P. and ROUBY J.J.: Comparative diagnostic performances of auscultation, chest radiography, and lung ultrasonography in acute respiratory distress syndrome. Anesthesiology: The Journal of the American Society of Anesthesiologists, 100 (1): 9-15, 2004.

6- LICHTENSTEIN D.A. and MEZIERE G.A.: Relevance of lung ultrasound in the diagnosis of acute respiratory failure*: The BLUE Protocol. Chest., 134 (1): 117-25, 2008.

7- RELLO J., LODE H., CORNAGLIA G. and MASTERTON R.: A European care bundle for prevention of ventilator-associated pneumonia. Intensive Care Medicine, 36 (5): 773-80, 2010.

8- MUKHTAR A., ZAGHLOL A., MANSOUR R., HASANIN A., EL-ADAWY A., MOHAMED H., et al.: Reduced incidence of methicillin-resistant Staphylococcus aureus ventilator-associated pneumonia in trauma patients: A new insight into the efficacy of the ventilator care bundle. Trauma, 16 (3): 202-6, 2014.

9- CARLIER M., ROBERTS J.A., STOVE V., VERSTRAETE A.G., LIPMAN J. and De WAELE J.J.: A simulation study reveals lack of pharmacokinetic/pharmacodynamic target attainment in de-escalated antibiotic therapy in critically ill patients. Antimicrobial agents and chemotherapy, 59 (8): 4689-94, 2015.

10- KNAAK E., CAVALIERI S.J., ELSASSER G.N., PREHEIM L.C., GONITZKE A. and DESTACHE C.J.: Does antibiotic de-escalation for nosocomial pneumonia impact Intensive Care Unit length of stay? Infectious Diseases in Clinical Practice, 21 (3): 172-6, 2013.

11- DELLINGER R.P., LEVY M.M., CARLET J.M., BION J., PARKER M.M., JAESCHKE R., et al.: Surviving Sepsis Campaign: International guidelines for management 
of severe sepsis and septic shock: 2008. Intensive Care Medicine, 34 (1): 17-60, 2008.

12- MONGODI S., VIA G., GIRARD M., ROUQUETTE I., MISSET B., BRASCHI A., et al.: Lung ultrasound for early diagnosis of ventilator-associated pneumonia. Chest, 149 (4): 969-80, 2016.

13- CORTELLARO F., COLOMBO S., COEN D. and DUCA P.G.: Lung ultrasound is an accurate diagnostic tool for the diagnosis of pneumonia in the Emergency Department. Emerg. Med. J., 29 (1): 19-23, 2012.

14- LICHTENSTEIN D., MEZIÈRE G. and SEITZ J.: The dynamic air bronchogram: A lung ultrasound sign of alveolar consolidation ruling out atelectasis. Chest, 135 (6): 1421-5, 2009.

15- BERLET T., ETTER R., FEHR T., BERGER D., SENDI P. and MERZ T.M.: Sonographic patterns of lung consolidation in mechanically ventilated patients with and without ventilator-associated pneumonia: A prospective cohort study. Journal of Critical Care, 30 (2): 327-33, 2015.
16- CORRADI F., BALL L., BRUSASCO C., VARGAS M., GARLASCHI A., ALTOMONTE F., et al.: Editors. Lung ultrasonography fails detection of non-subpleural community acquired pneumonia. Intensive Care Medicine, Springer 233 Spring ST, New York, NY 10013 USA, 2012.

17- GATT M.E., SPECTRE G., PALTIEL O., HILLER N. and STALNIKOWICZ R.: Chest radiographs in the Emergency Department: Is the radiologist really necessary? Postgraduate Medical Journal, 79 (930): 214-7, 2003.

18- ZAGLI G., COZZOLINO M., TERRENI A., BIAGIOLI T., CALDINI A.L. and PERIS A.: Diagnosis of ventilatorassociated pneumonia: A pilot, exploratory analysis of a new score based on procalcitonin and chest echography. Chest, 146 (6): 1578-85, 2014.

19- XIROUCHAKI N., MAGKANAS E., VAPORIDI K., KONDILI E., PLATAKI M., PATRIANAKOS A., et al.: Lung ultrasound in critically ill patients: Comparison with bedside chest radiography. Intensive Care Med., 37 (9): 1488-93, 2011.

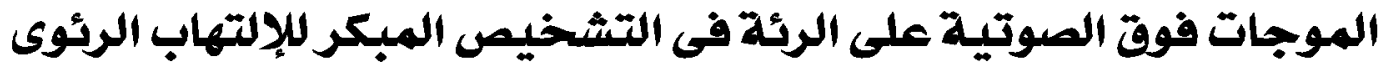 المصاحب لجهاز التنفس الصناعى التهى الميكر}

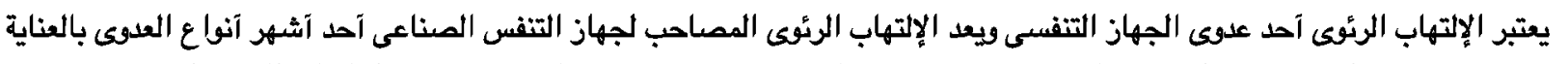

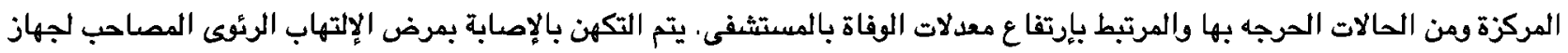

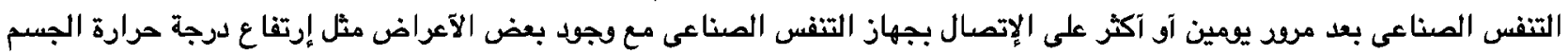

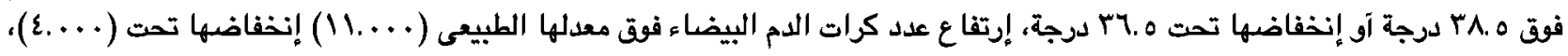

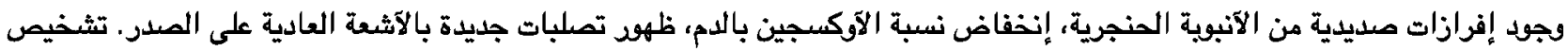

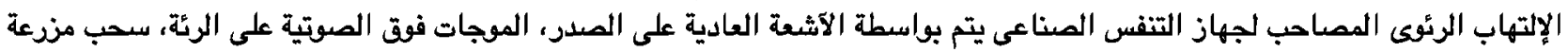

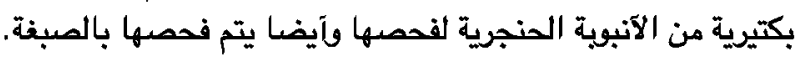

الهدف من الدراسة: لراسة دود الموجات فوق الصوتية على الرئة فى التثخيص المبكر للإلتهاب الرئوى المصاحب لجهاز التنفس الصناعى مقارنة بالآثعة العادية على المدر.

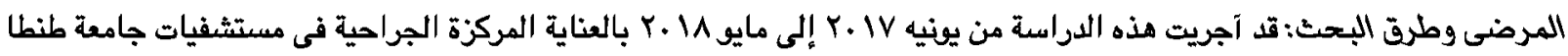

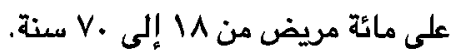

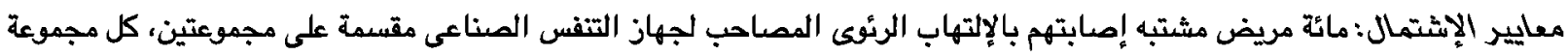

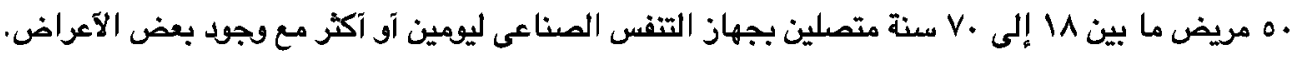

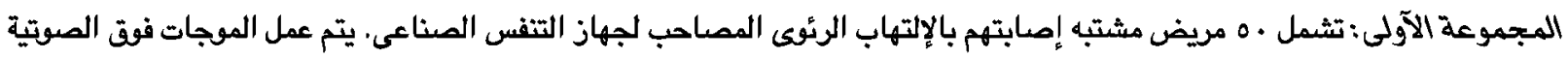

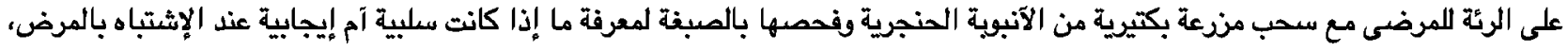

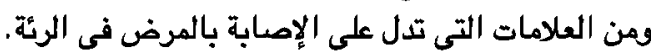
• • تصلبات صغيرة تحت الغشاء البلودى.

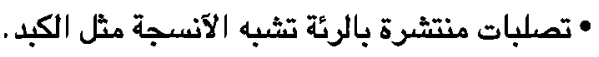
•تجمعات هوائية خطية آو متثبعة بداخل الشعب الهوائية تتحرك مع التنفس. 


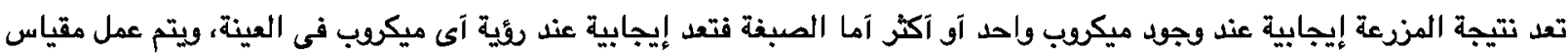
للموجات فوق الصوتية على الرئة فى الإلتهاب الرئوى المصاحب لجهاز ولئ التنفس الصناعى للمساعدة في التثخيص المبكر للمريض ويحسب • منطقتين آو آكثر من تصلبات صغيرة تحت الفشاء البلوىى تآخذ نقطة واحلة. • منطقة آو آكثر من تجمعات الهوائية بالشعب الهوائية تآخذ نقطتين. • إفرازات صديدية من الآنبوبة الحنجريبة تآخذ نقطة واحدة. يتم إدراج نتيجة المزرعة البكتيرية والفحص المباشر بالصبغة هع هذا المقياس وإذا كانت نتيجة الفحص المباشر بالصبغة آو المزرعة البكتيرية إيجابية تآخذ نقطتين.

المجهوعة الثانية: تشمل •0 مريض، يتم عمل آشعة عادية على الصدر لهم مع سحب مزرعة بكتيرية من الآنبوبة الحنجرية وفحصها

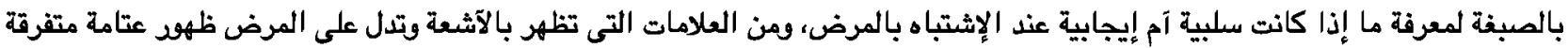

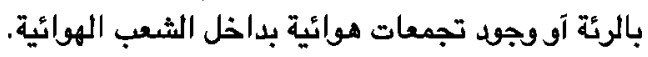
النتائج: تمت جدولة النتائج وتحليلها إحصائيا وقد وجد آنه: • لا يوجد فرق ذو دلالة إحصائية فيما يتعلق بالييانات الديموغرافية بالمجموعتين مثل العمر، النوع والونن.

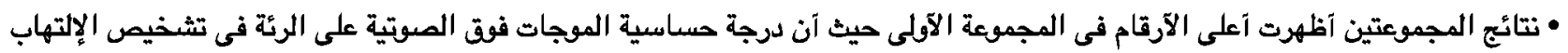

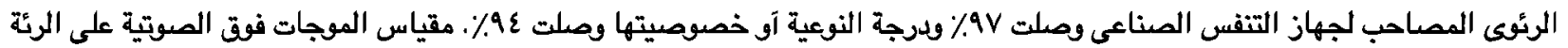

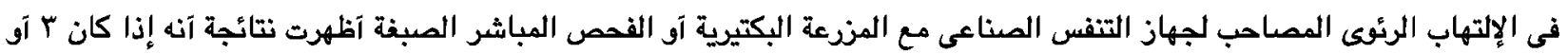

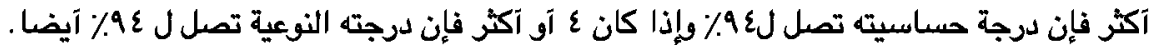

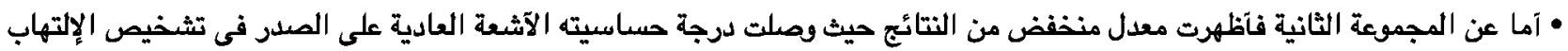

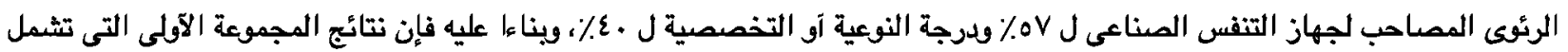

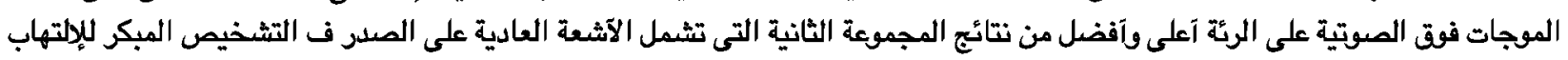
الرئوى المصاحب لجهاز التنفس الصناعى.

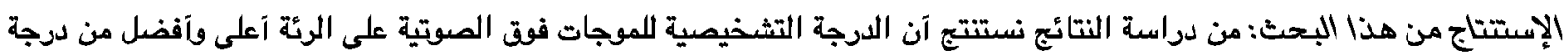

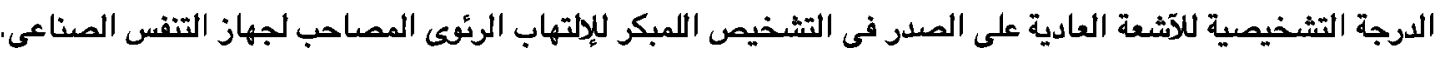

